\title{
Use of Near Infrared Spectroscopy to Asses Remote Ischemic Preconditioning in Skeletal Muscle
}

\author{
Jana Ambrozic, ${ }^{1}$ Mitja Lainscak, ${ }^{2,3}$ and Matej Podbregar ${ }^{4}$ \\ ${ }^{1}$ Department of Cardiology, University Medical Centre Ljubljana, 1000 Ljubljana, Slovenia \\ ${ }^{2}$ Division of Cardiology, University Clinic of Respiratory and Allergic Diseases Golnik, 4204 Golnik, Slovenia \\ ${ }^{3}$ Applied Cachexia Research, Department of Cardiology, Charité, Campus Virchow-Klinikum, 13353 Berlin, Germany \\ ${ }^{4}$ Department of Intensive Care Medicine, University Medical Centre Ljubljana, 1000 Ljubljana, Slovenia
}

Correspondence should be addressed to Matej Podbregar; matej.podbregar@guest.arnes.si

Received 1 November 2012; Revised 23 January 2013; Accepted 23 January 2013

Academic Editor: Michael S. Wolin

Copyright (C) 2013 Jana Ambrozic et al. This is an open access article distributed under the Creative Commons Attribution License, which permits unrestricted use, distribution, and reproduction in any medium, provided the original work is properly cited.

\begin{abstract}
Remote ischemic preconditioning (IPC) is a procedure during which brief periods of ischemia protect distant organ from ischemiareperfusion injury. Appling IPC on an upper arm, this phenomenon has been demonstrated in several studies. Skeletal muscle tissue oxygenation at rest $\left(\mathrm{StO}_{2}\right)$ and $\mathrm{StO}_{2}$ deoxygenation rate during vascular occlusion can be measured using near infrared spectroscopy (NIRS). We aimed to investigate the effects of remote upper arm IPC on $\mathrm{StO}_{2}$ and flow-mediated dilatation (FMD) in healthy male volunteers. In a randomized controlled crossover trial, resting $\mathrm{StO}_{2}, \mathrm{StO}_{2}$ deoxygenation rate, and FMD were measured on testing arm at baseline and after 60 minutes. After basal measurements IPC protocol on a contralateral arm was performed. StO ${ }_{2}$ deoxygenation rate was significantly lower after remote, the IPC cycles in comparison to deoxygenation rate at baseline $(9.7 \pm 2.6$ versus $7.5 \pm 2.5 \%, P=0.002)$. Comparison of deoxygenation rates showed a significant difference between the IPC and the control protocol $(F=5.512, P=0.003)$. No differences were observed in FMD before and after remote IPC and in the control protocol. In healthy young adults, remote IPC reduces $\mathrm{StO}_{2}$ deoxygenation rate but has no significant impact on FMD. NIRS technique offers a novel approach to asses skeletal muscle adaptation in response to remote ischemic stimuli.
\end{abstract}

\section{Introduction}

Ischemia-reperfusion injury is a systemic event resulting in damage to local and remote organs. Restoration of blood supply to an organ after a critical period of ischemia causes additional tissue damage and organ dysfunction leading to significant morbidity and mortality. It has been described in a number of clinical settings: acute coronary occlusion, cardiopulmonary bypass procedures, organ transplants, and shock [1-3]. Remote ischemic preconditioning (IPC) is a procedure where brief periods of ischemia in one tissue prior to sustained ischemia reduce ischemia-reperfusion injury in another remote tissue $[4,5]$. After early experiments in animals [6], both in vitro and in vivo studies suggested the significance of this protective effect in humans, particularly in the setting of myocardial ischemia [7-10]. However, there is still no agreement about standardized IPC protocol.
The precise mechanisms involved in IPC are not yet established. A possible pathway involves the generation of nitric oxide and an effect on endothelial function $[5,8]$. Molecular mechanism is not completely understood, but probably involves the released humoral mediators from ischemic tissue, including adenosine, bradykinin, and opioids, which react with the cell surface receptors and activate protein kinase cascades. The end-effectors may be mitochondrial ATP-dependent potassium channels and mitochondrial permeability transition pores, whose opening during ischemia may be lethal [11-14].

Skeletal muscle has been investigated as a remote stimulus for either distant skeletal muscle or cardiac protection in several studies $[9,15-18]$. Ischemic conditioning of the skeletal muscle on an upper or lower limb is a noninvasive, readily reproducible method for studying the effects on other 
remote organs and, therefore, might be easily translated into clinical practice.

Near infrared spectroscopy (NIRS) has been used to assess peripheral microvascular function in a variety of clinical settings [19-23]. Illuminating an infrared band of 680$800 \mathrm{~nm}$ NIRS exploits the difference in absorption spectra between the oxygenated and deoxygenated hemoglobin and partly myoglobin and thus assesses the balance between local arterial supply and cellular oxygen consumption within the muscle tissue. NIRS enables continuous monitoring of skeletal muscle tissue oxygenation $\left(\mathrm{StO}_{2}\right)$, which reflects this balance between oxygen delivery and consumption. With the stagnant ischemia provoked by vascular occlusion test (VOT), dynamic changes in $\mathrm{StO}_{2}$ and thus microvascular function/tissue metabolism can be followed and evaluated [24].

To the best of our knowledge, no reports about the effect of remote IPC on dynamic changes in $\mathrm{StO}_{2}$ of skeletal muscle, measured by NIRS are available. In previous studies, positive effects of IPC on skeletal muscle were shown as prevention of the endothelial dysfunction and systemic neutrophil activation $[8,12,17,18]$.

The aim of our study was to evaluate the effect of remote IPC on skeletal muscle $\mathrm{StO}_{2}$ using NIRS technique. Considering the ischemia-induced deoxygenation rate of the tissue oxygenation curve as a surrogate for tissue oxygen consumption, we hypothesized that this technique might detect the ability of skeletal muscle adaptation to remote ischemic stimuli applied in the contralateral arm. We also evaluated the effect of remote IPC on endothelial function using flow-mediated dilatation method (FMD).

\section{Materials and Methods}

2.1. Study Design. The study was designed as a randomized controlled crossover trial. The study protocol was approved by the National Ethics Committee of Slovenia.

2.2. Subjects. Fourteen male healthy volunteers with no significant past medical history were enrolled in the study. Exclusion criteria were any chronic illness, medication, and smoking. The subjects were investigated in two experiments. They were randomly assigned to the remote IPC or the control protocol and were then crossed over to another protocol after at least one week in a random sequence. The two experiments for each subject were performed in the morning after an overnight fast and after 10 minutes of rest in supine position. The selected testing arm was the left, nondominant arm in all subjects. All participants gave written informed consent before any study-related procedure.

2.3. Skeletal Muscle Near Infrared Spectroscopy and Vascular Occlusion Test. $\mathrm{StO}_{2}$ was measured by NIRS using the InSpectra 325 tissue oxygenation monitor (Hutchinson Technology Inc., West Highland Park Drive NE, MN, USA), which measures tissue absorbance values between 650 and $900 \mathrm{~nm}$. The tissue absorbance values (four wavelengths data) are transformed into scaled second derivative absorbance values to provide a tissue spectral measurement that is robust to total hemoglobin and optical path length changes $[25,26]$. $25 \mathrm{~mm}$ InSpectra probe (source-detector separation $25 \mathrm{~mm}$, penetration depth $23 \mathrm{~mm}$, and $95 \%$ signal threshold) was used $[22,23]$. Probe was moved over the thenar prominence in order to localize the maximum $\mathrm{StO}_{2}$ and then fixed with InSpectra Shield. $\mathrm{StO}_{2}$ data was monitored and stored onto a computer using InSpectra software.

After measuring resting $\mathrm{StO}_{2}$ for 30 seconds, VOT was performed as described earlier [22, 23]. In short, testing arm was subjected to ischemia by inflating a blood pressure tourniquet, placed around the forearm, to a pressure of $250 \mathrm{mmHg}$ for 3 minutes and then rapidly released. $\mathrm{StO}_{2}$ was continuously measured over the thenar muscle of the testing arm before, during and 5 minutes after VOT. All VOTs were done using the same blood pressure tourniquet.

The dynamic $\mathrm{StO}_{2}$ curve parameters reported in this study are resting $\mathrm{StO}_{2}(\%), \mathrm{StO}_{2}$ deoxygenation rate (\%/min), which was measured during vascular occlusion, $\mathrm{StO}_{2}$ reoxygenation rate $(\% / \mathrm{min})$, and maximal $\mathrm{StO}_{2}(\%)$, measured after release of vascular occlusion. InSpectra analysis software 2.0 was used to automatically calculate $\mathrm{StO}_{2}$ slope gradient.

2.4. Flow-Mediated Dilatation. Endothelial function was assessed by measuring FMD of the brachial artery using a Vivid 4 ultrasound machine (GE Medical Systems, USA) with a $7 \mathrm{MHz}$ linear array transducer as described [27]. The brachial artery was scanned in the longitudinal section 2$15 \mathrm{~cm}$ above the elbow finding the clearest images of the anterior and posterior wall layers. The mean arterial diameter was measured at the end of diastole, which was determined by simultaneous monitoring of the electrocardiogram. At least three cardiac cycles were analyzed for each scan and the measurements averaged. The flow velocity was measured at a fixed incident angle of $60^{\circ}$ to the vessel with the range gate of $1.5 \mathrm{~mm}$ located in the centre of the artery. The basal flow was estimated by multiplying the velocity time integral of the Doppler flow signal (corrected for incident angle) by the vessel cross-sectional area. Flow after VOT was recorded within the first 15 seconds and diameter measurements were taken 30-90 seconds after cuff deflation. FMD was expressed as the percentage change of the diameter after reactive hyperemia relative to the basal diameter. Ultrasound data included basal diameter of the brachial artery $(\mathrm{mm})$, basal flow ( $\mathrm{mL} / \mathrm{min})$, flow after AOT (mL/min), and FMD (\%).

2.5. IPC Protocol. The subjects rested in the supine position for 10 minutes before measurements. Baseline NIRS measurements were performed before and during the first VOT on a testing arm. FMD was assessed according to the changes in arterial diameter before and after VOT. After baseline measurements, remote IPC was applied by three cycles of 5 minutes of ischemia and 5 minutes of reperfusion on the contralateral upper arm. Ischemia was induced by inflating a blood pressure tourniquet around the upper arm to a pressure $50 \mathrm{mmHg}$ above the subject's resting systolic blood pressure. After completion of ischemia and reperfusion cycles, the subjects rested for another 30 minutes. The second NIRS 


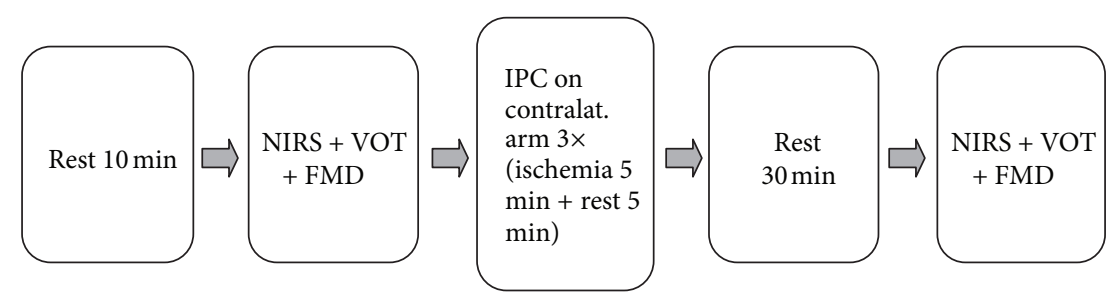

(a) Ischemic preconditioning protocol

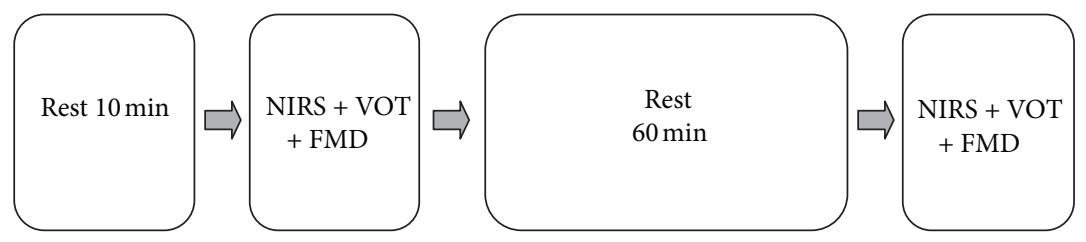

(b) Control protocol

FIGURE 1: Flow chart of the experiment. NIRS: near infrared spectroscopy; VOT: vascular occlusion test; FMD: flow-mediated dilatation; IPC: ischemic preconditioning.

measurements and VOT were then performed on a testing arm in the same way, 60 minutes after baseline measurements and FMD was assessed.

2.6. Control Protocol. The subjects rested in the supine position for 10 minutes before measurements. Baseline NIRS measurements were performed before and during the first VOT on a testing arm and FMD was assessed. Following 60 minutes of rest, the second VOT with NIRS and FMD measurements were performed. The protocols are schematically illustrated in Figure 1.

2.7. Data Analysis. Data is presented as mean \pm standard deviation (SD) or mean (range). Data were analyzed using the Statistical Package for the Social Sciences (SPSS, version 10.0, SPPS Inc., Chicago, IL, USA). Skeletal muscle $\mathrm{StO}_{2}$ data in the IPC and the control protocol were compared using One way repeated measures analysis of variance with all pairwise multiple comparison procedure by Holm-Sidak method. $P$ values $<0.05$ were regarded as significant. In individual subject, ultrasound data in the IPC and the control protocol were compared using paired samples Student's $t$-test with $P$ values $<0.05$ regarded as significant.

\section{Results}

A total of fourteen male healthy volunteers with mean (range) age of 32 (26-47) years, body weight 76 (57-105) kg, height 181 $(162-204) \mathrm{cm}$, body mass index 25.3 (21.7-28.4), and systolic blood pressure $110(100-155) \mathrm{mmHg}$ participated in the study.

There was no difference in resting $\mathrm{StO}_{2}$ at baseline and after 60 minutes in the IPC protocol $(85 \pm 2 \%$ versus $85 \pm 2 \%$, $P=0.9)$, nor in the control protocol ( $85 \pm 2 \%$ versus $85 \pm 2 \%$, $P=0.9)$. There was also no difference in resting $\mathrm{StO}_{2}$ between both protocols $(P$ between groups $=0.93$ ).

Compared to baseline $\mathrm{StO}_{2}$ deoxygenation rate was significantly lower after the IPC protocol $(9.7 \pm 2.6 \% / \mathrm{min}$ versus
$7.5 \pm 2.5 \% / \mathrm{min}, P=0.002) . \mathrm{StO}_{2}$ deoxygenation rates in the control protocol were found to be similar compared to baseline $(10.0 \pm 3.4 \% / \mathrm{min}$ versus $9.5 \pm 4.0 \% / \mathrm{min}, P=$ 0.4). Comparison of $\mathrm{StO}_{2}$ deoxygenation rates showed a statistically significant difference between the IPC and the control protocol $(F=5.512, P=0.003)$. A graphical representation of skeletal muscle $\mathrm{StO}_{2}$ during VOT in both protocols in an individual subject is shown in Figure 2. Individual data of skeletal muscle $\mathrm{StO}_{2}$ deoxygenation of the study population is shown Figure 3. There was no difference in basal $\mathrm{StO}_{2}$, reoxygenation rate, and maximal $\mathrm{StO}_{2}$ in all study analyses (Table 1).

No differences were observed in the basal diameter of the brachial artery, basal flow, flow after VOT, and FMD before and after IPC in the IPC protocol and in the control protocol. There were no differences between both protocols (Table 2).

\section{Discussion}

Our study investigated the ischemic adaptation of skeletal muscle induced by IPC using NIRS methodology in healthy subjects. After remote ischemic cycles, we confirmed reduced $\mathrm{StO}_{2}$ deoxygenation rate during vascular occlusion, demonstrating reduced muscle oxygen consumption. Current study confirmed the usefulness of NIRS technology to asses remote IPC in skeletal muscle.

IPC by ischemic cycles applied on skeletal muscle was tested in a variety of clinical studies, which proved that remote IPC has beneficial effect on distant, treated organs. Greatly the studies were focused on IPC of the heart since cardiovascular diseases account nowadays for significant morbidity and mortality. The beneficial effect of IPC was first studied in children undergoing surgery for congenital heart defects that showed lower troponin levels and less inotropic requirement if the patients were subjected to IPC [16]. In adult patients who underwent elective coronary artery bypass graft surgery, remote IPC consisting of ischemic cycles of the upper arm resulted in a significant reduction 


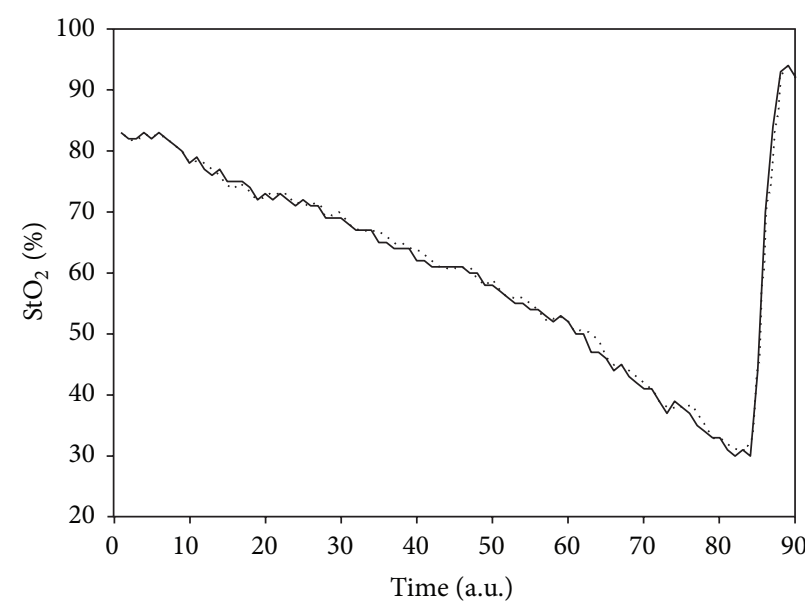

- Control before …. Control after

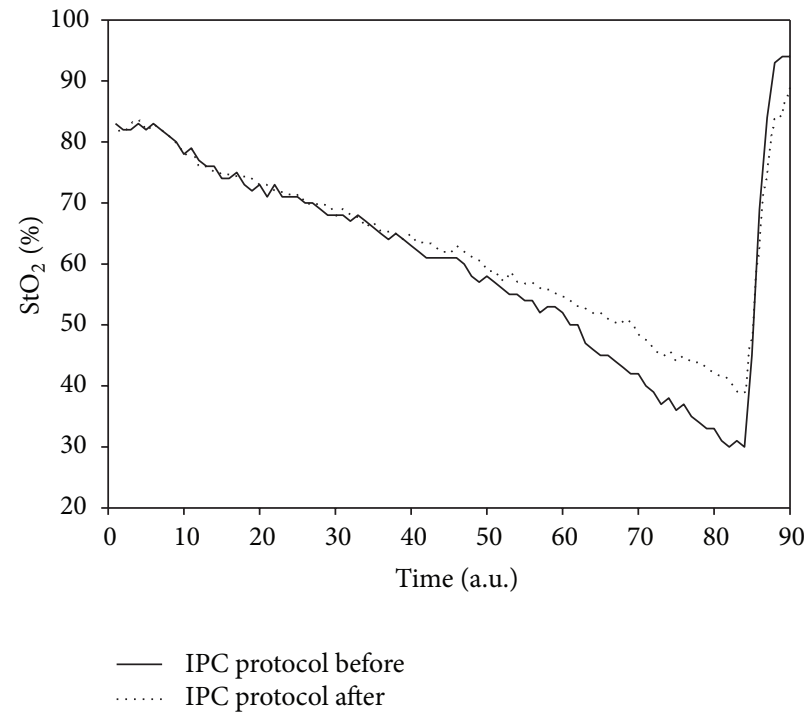

(b)

FIgURE 2: Skeletal muscle $\mathrm{StO}_{2}$ during vascular occlusion test in both experiments in an individual subject. Control before: skeletal muscle $\mathrm{StO}_{2}$ in the control protocol at baseline; control after: skeletal muscle $\mathrm{StO}_{2}$ in the control protocol after 60 minutes of rest (at the end of the protocol); IPC protocol before: skeletal muscle $\mathrm{StO}_{2}$ in the ischemic preconditioning protocol at baseline; IPC protocol after: skeletal muscle ischemic preconditioning in the ischemic preconditioning protocol at the end of the protocol.

TABLE 1: Skeletal muscle tissue oxygenation $\left(\mathrm{StO}_{2}\right)$ during vascular occlusion test.

\begin{tabular}{|c|c|c|c|c|c|c|c|}
\hline \multirow{2}{*}{ Variable } & \multicolumn{3}{|c|}{ IPC protocol $(n=14)$} & \multicolumn{3}{|c|}{ Control $(n=14)$} & \multirow{2}{*}{ Statistics between treatments } \\
\hline & Before & After & Statistics & Before & After & Statistics & \\
\hline Basal $\mathrm{StO}_{2}(\%)$ & $85 \pm 2$ & $85 \pm 2$ & ns & $85 \pm 3$ & $84 \pm 4$ & ns & 0.934 \\
\hline Deoxygenation rate $(\% / \mathrm{min})$ & $9.7 \pm 2.6$ & $7.5 \pm 2.5$ & 0.002 & $9.5 \pm 4.0$ & $10.0 \pm 3.4$ & 0.4 & 0.003 \\
\hline Reoxygenation rate $(\% / \mathrm{min})$ & $230 \pm 72$ & $230 \pm 100$ & ns & $232 \pm 123$ & $250 \pm 113$ & ns & 0.922 \\
\hline Maximal StO $2(\%)$ & $94 \pm 2$ & $94 \pm 1$ & ns & 95 & 95 & ns & 0.955 \\
\hline
\end{tabular}

IPC protocol before: skeletal muscle $\mathrm{StO}_{2}$ data in the ischemic preconditioning protocol at baseline; IPC protocol after: skeletal muscle $\mathrm{StO}_{2}$ data in the ischemic preconditioning protocol at the end of the protocol; control before: skeletal muscle $\mathrm{StO}_{2}$ data in the control protocol at baseline; control after: skeletal muscle $\mathrm{StO}_{2}$ data in the control protocol after 60 minutes of rest; and ns: nonsignificant.

in troponin release following surgery [9]. Even in a large group of patients undergoing elective percutaneous coronary intervention, those who were treated by remote upper arm IPC were more likely to have undetectable troponin release following intervention and better morbidity results at 6 months [28]. In patients presenting with acute myocardial infarction, remote IPC by repeated limb ischemia before hospital admission increased myocardial salvage measured by myocardial perfusion imaging [15].

NIRS technology has been used as a noninvasive, readily accessible method for assessing tissue microcirculation in a number of different research and clinical settings. In response to ischemia, it also allows evaluation of dynamic changes in microcirculation. Using NIRS, the changes in skeletal muscle oxygenation have been demonstrated in chronic heart failure patients at rest and during exercise [20, 29], in patients undergoing haemodialysis [21], during abdominal aortic and cardiac surgery $[30,31]$, and in patients with peripheral arterial disease [32], cirrhosis [33], septic shock $[22,23]$, and metabolic myopathies [34]. NIRS has been already applied to demonstrate the effect of IPC on changes in myocardial oxygenation in dogs; IPC has been induced by repeated periods of coronary occlusion [35]. No previous study, however, utilized NIRS to demonstrate the effect of remote IPC on skeletal muscle in humans.

The question remains what the underlying mechanism of the decreased deoxygenation rate is. In animal studies, it has been shown that ischemia-reperfusion injury alters the mitochondrial oxidative phosphorylation and that IPC preserves mitochondrial enzyme activities [36]. So we can speculate that decreased deoxygenation rate demonstrated in our study reflects certain degree of beneficial tissue adaptation on remote ischemic conditions, possibly through modification of mitochondrial oxygen metabolism. However, NIRS does not measure mitochondrial function and other explanations for decreased deoxygenation rate should be considered. The rate of postischemic vasodilatation might be enhanced in response to IPC, but there were no differences in reoxygenation rate and maximal $\mathrm{StO}_{2}$ between the control and the IPC protocols in our study. There were also 


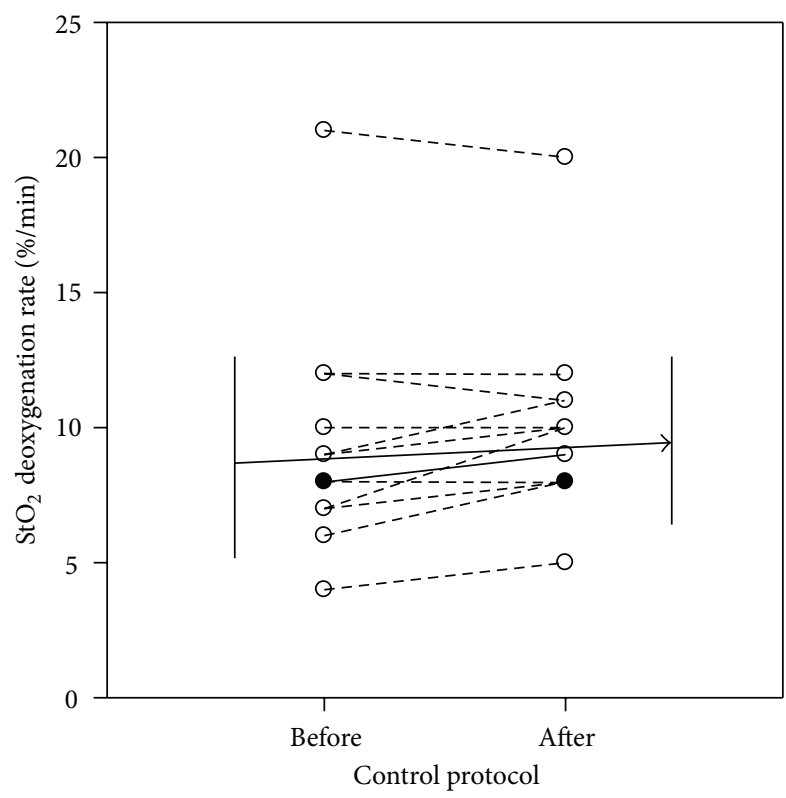

(a)

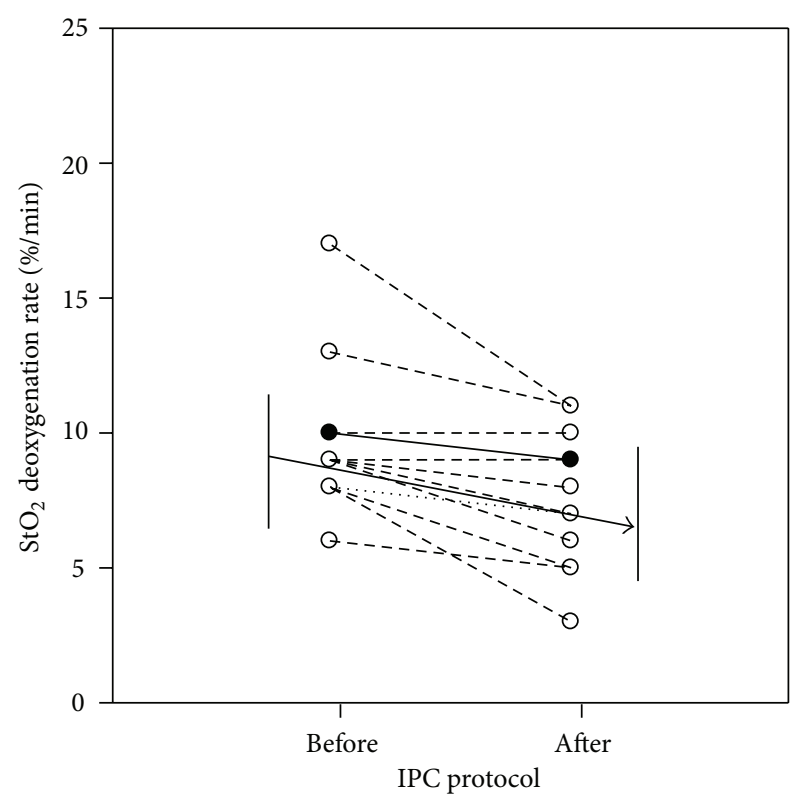

(b)

FIGURE 3: Individual data of skeletal muscle $\mathrm{StO}_{2}$ deoxygenation of the study population in the ischemic preconditioning (IPC) and the control protocol. Compared to baseline, $\mathrm{StO}_{2}$ deoxygenation rate was significantly lower after the IPC protocol $(9.7 \pm 2.6 \% / \mathrm{min}$ versus $7.5 \pm$ $2.5 \% / \mathrm{min}, P=0.002)$. $\mathrm{StO}_{2}$ deoxygenation rates in the control protocol were found to be similar compared to baseline $(10.0 \pm 3.4 \% / \mathrm{min}$ versus $9.5 \pm 4.0 \% / \mathrm{min}, P=0.4)$. Comparison of $\mathrm{StO}_{2}$ deoxygenation rates showed a statistically significant difference between the IPC and the control protocol $(F=5.512, P=0.003)$. In the figure, there are less lines that were the actual number of the study participants $(n=14)$ because three pairs of data in the control and two pairs of data in the IPC protocol were the same (black points).

TABLE 2: Flow-mediated dilatation-ultrasound data.

\begin{tabular}{lccccccc}
\hline \multirow{2}{*}{ Variable } & \multicolumn{3}{c}{ IPC protocol $(n=14)$} & \multicolumn{3}{c}{ Control $(n=14)$} & \multicolumn{2}{c}{ Statistics between treatments } \\
& Before & After & Statistics & Before & After & Statistics & \\
\hline Brachial artery diameter $(\mathrm{mm})$ & $4.3 \pm 4$ & $4.2 \pm 3$ & $\mathrm{~ns}$ & $4.1 \pm 2$ & $4.2 \pm 3$ & $\mathrm{~ns}$ & 0.226 \\
Basal flow (mL/min) & $157 \pm 81$ & $175 \pm 51$ & $\mathrm{~ns}$ & $169 \pm 62$ & $163 \pm 57$ & $\mathrm{~ns}$ & 0.788 \\
Flow after VOT (mL/min) & $902 \pm 384$ & $937 \pm 373$ & $\mathrm{~ns}$ & $930 \pm 326$ & $900 \pm 233$ & $\mathrm{~ns}$ & 0.929 \\
FMD (\%) & $9.4 \pm 3.8$ & $9.5 \pm 3.8$ & $\mathrm{~ns}$ & $9.9 \pm 3.4$ & $9.9 \pm 3.4$ & $\mathrm{~ns}$ & 0.941 \\
\hline
\end{tabular}

IPC protocol before: ultrasound data in the ischemic preconditioning protocol at baseline; IPC protocol after: ultrasound data in the ischemic preconditioning protocol at the end of the protocol; control before: ultrasound data in the control protocol at baseline; control after: ultrasound data in the control protocol at the end of the protocol; VOT: vascular occlusion test; FMD: flow-mediated dilatation; and ns: nonsignificant.

no changes in FMD; therefore we could not identify the modulation of oxygen delivery. According to our data, we can conclude that IPC induces tissue metabolic changes instead of changes of blood flow. However, 5 minutes of ischemic time that we used were relatively short in comparison to other studies where 20 minutes of ischemia were applied [8]. This might be the reason why we could not demonstrate any differences in FMD in our experiments, not even reduced FMD after VOT, which was possibly too short in duration to cause measurable effects. Our conclusions are similar to the findings of Kilian et al. [37], who otherwise confirmed a significantly reduced FMD after 10 minutes of ischemia but failed to reproduce its prevention by remote IPC using shorter IPC stimuli and shorter time delay between stimuli and ischemic event.

Major limitation of our study is that the NIRS device that was used does not provide absolute concentrations of oxyhemoglobin, deoxyhemoglobin, and total hemoglobin; so we were not able to calculate oxygen consumption as proposed in previous studies either by arterial or venous occlusions [38-40]. Venous occlusion is preferable to arterial occlusion because the procedure is less inconvenient for the subjects and can be repeated at short time intervals. However, venous occlusion is more prone to variations in flow within the arm due to the changes in blood pressure and local vasoreactivity, whereas these influences are negligible during arterial occlusion that characterizes closed compartment, temporary cut-off from centrally mediated variations. It seems that arterial occlusion method has higher reproducibility as compared to venous occlusion [39]. Calculations of the skeletal muscle oxygen consumption are prone to errors due to changes in the optical properties of the tissue and can be expressed in milliliters of oxygen per minute per 100 grams only after assuming tissue density. Absolute values of 
the skeletal muscle oxygen consumption are clinically not important; that is why we decided to monitor only tissue desaturation (deoxygenation rate), which is a surrogate of oxygen consumption.

\section{Conclusions}

Our findings confirm that remote ischemic cycles provide adaptation of skeletal muscle in terms of decrease oxygen consumption when exposed to ischemia that was demonstrated by NIRS technology. In spite of its recognized powerful effect, the precise mechanism of this phenomenon is still unknown. There are several factors influencing the extent of IPC effects. The length and magnitude of the ischemic event as well as the intensity of remote ischemic stimuli seem to play a role. It has been recognized that aging and other cardiovascular risk factors, such as diabetes, smoking, hyperlipidemia, hypertension, and obesity, are associated with lower protective effect of IPC, but the threshold for protection is unclear [41]. Skeletal muscle NIRS during vascular occlusion test can be applied to quantitatively determine the effectiveness of IPC. In accordance with our findings, we could propose NIRS to test various preconditioning protocols with ischemic or even pharmacological stimuli in a different population of patients who might benefit from preconditioning therapeutic strategy with the aim to find individual threshold for protection. Further studies would be necessary to confirm these assumptions.

\section{Acknowledgment}

This work was supported by Research Program P3-0043, Slovenian Research Agency. The authors thank all subjects, doctors, nurses, and technical support staff for their assistance in this study. They declare no conflict of interests.

\section{References}

[1] G. Ambrosio and I. Tritto, "Reperfusion injury: experimental evidence and clinical implications," American Heart Journal, vol. 138, no. 2, pp. S69-S75, 1999.

[2] M. A. Daemen, B. de Vries, and W. A. Buurman, "Apoptosis and inflammation in renal reperfusion injury," Transplantation, vol. 73, no. 11, pp. 1693-1700, 2002.

[3] B. E. Miller and J. H. Levy, "The inflammation response to cardiopulmonary bypass," Journal of Cardiothoracic and Vascular Anesthesia, vol. 11, no. 3, pp. 355-366, 1997.

[4] R. Ferrari, C. Ceconi, S. Curello, G. Percoco, T. Toselli, and G. Antonioli, "Ischemic preconditioning, myocardial stunning, and hibernation: basic aspects," American Heart Journal, vol. 138, no. 2, pp. S61-S68, 1999.

[5] K. Laude, P. Beauchamp, C. Thuillez, and V. Richard, "Endothelial protective effects of preconditioning," Cardiovascular Research, vol. 55, no. 3, pp. 466-473, 2002.

[6] K. Przyklenk, B. Bauer, M. Ovize, R. A. Kloner, and P. Whittaker, "Regional ischemic "preconditioning" protects remote virgin myocardium from subsequent sustained coronary occlusion," Circulation, vol. 87, no. 3, pp. 893-899, 1993.
[7] B. Günaydin, I. Cakici, H. Soncul et al., "Does remote organ ischaemia trigger cardiac preconditioning during coronary artery surgery?" Pharmacological Research, vol. 41, no. 4, pp. 493-496, 2000.

[8] R. K. Kharbanda, M. Peters, B. Walton et al., "Ischemic preconditioning prevents endothelial injury and systemic neutrophil activation during ischemia-reperfusion in humans in vivo," Circulation, vol. 103, no. 12, pp. 1624-1630, 2001.

[9] V. Venugopal, D. J. Hausenloy, A. Ludman et al., "Remote ischaemic preconditioning reduces myocardial injury in patients undergoing cardiac surgery with cold-blood cardioplegia: a randomised controlled trial," Heart, vol. 95, no. 19, pp. 1567-1571, 2009.

[10] Z. K. Wu, T. Iivainen, E. Pehkonen, J. Laurikka, and M. R. Tarkka, "Ischemic preconditioning suppresses ventricular tachyarrhythmias after myocardial revascularization," Circulation, vol. 106, no. 24, pp. 3091-3096, 2002.

[11] A. D. T. Costa, S. V. Pierre, M. V. Cohen, J. M. Downey, and K. D. Garlid, "cGMP signalling in pre- and post-conditioning: the role of mitochondria," Cardiovascular Research, vol. 77, no. 2, pp. 344-352, 2008.

[12] S. P. Loukogeorgakis, R. Williams, A. T. Panagiotidou et al., "Transient limb ischemia induces remote preconditioning and remote postconditioning in humans by a KATP channeldependent mechanism," Circulation, vol. 116, no. 12, pp. 13861395, 2007.

[13] S. R. Walsh, T. Tang, U. Sadat, D. P. Dutka, and M. E. Gaunt, "Cardioprotection by remote ischaemic preconditioning," British Journal of Anaesthesia, vol. 99, no. 5, pp. 611-616, 2007.

[14] D. M. Yellon and J. M. Downey, "Preconditioning the myocardium: from cellular physiology to clinical cardiology," Physiological Reviews, vol. 83, no. 4, pp. 1113-1151, 2003.

[15] H. E. Bøtker, R. Kharbanda, M. R. Schmidt et al., "Remote ischaemic conditioning before hospital admission, as a complement to angioplasty, and effect on myocardial salvage in patients with acute myocardial infarction: a randomised trial," The Lancet, vol. 375, no. 9716, pp. 727-734, 2010.

[16] M. M. H. Cheung, R. K. Kharbanda, I. E. Konstantinov et al., "Randomized controlled trial of the effects of remote ischemic preconditioning on children undergoing cardiac surgery: first clinical application in humans," Journal of the American College of Cardiology, vol. 47, no. 11, pp. 2277-2282, 2006.

[17] R. K. Kharbanda, U. M. Mortensen, P. A. White et al., “Transient limb ischemia induces remote ischemic preconditioning in vivo," Circulation, vol. 106, no. 23, pp. 2881-2883, 2002.

[18] S. P. Loukogeorgakis, A. T. Panagiotidou, D. M. Yellon, J. E. Deanfield, and R. J. MacAllister, "Postconditioning protects against endothelial ischemia-reperfusion injury in the human forearm," Circulation, vol. 113, no. 7, pp. 1015-1019, 2006.

[19] K. Abozguia, T. T. Phan, G. N. Shivu et al., "Reduced in vivo skeletal muscle oxygen consumption in patients with chronic heart failure-a study using near infrared spectroscopy (NIRS)," European Journal of Heart Failure, vol. 10, no. 7, pp. 652-657, 2008.

[20] R. Belardinelli, "Monitoring skeletal muscle oxygenation during exercise by near infrared spectroscopy in chronic heart failure," Congestive Heart Failure, vol. 5, no. 3, pp. 116-124, 1999.

[21] R. A. De Blasi, R. Luciani, G. Punzo et al., "Microcirculatory changes and skeletal muscle oxygenation measured at rest by non-infrared spectroscopy in patients with and without 
diabetes undergoing haemodialysis," Critical Care, vol. 13, supplement 5, p. S9, 2009.

[22] R. Parežnik, R. Knezevic, G. Voga, and M. Podbregar, "Changes in muscle tissue oxygenation during stagnant ischemia in septic patients," Intensive Care Medicine, vol. 32, no. 1, pp. 87-92, 2006.

[23] M. Podbregar and H. Možina, "Skeletal muscle oxygen saturation does not estimate mixed venous oxygen saturation in patients with severe left heart failure and additional severe sepsis or septic shock," Critical Care, vol. 11, article R6, 2007.

[24] S. Nioka, R. Kime, U. Sunar et al., "A novel method to measure regional muscle blood flow continuously using NIRS kinetics information," Dynamic Medicine, vol. 5, article 5, 2006.

[25] D. E. Myers, L. D. Anderson, R. P. Seifert et al., "Noninvasive method for measuring local hemoglobin oxygen saturation in tissue using wide gap second derivative near-infrared spectroscopy," Journal of Biomedical Optics, vol. 10, no. 3, Article ID 034017, 2005.

[26] Y. Wickramasinghe and S. A. Spencer, "Quantification of peripheral oxygen consumption by near infrared spectroscopy," Archives of Disease in Childhood, vol. 89, no. 5, pp. F469-F470, 2004.

[27] M. Stout, "Flow-mediated dilatation: a review of techniques and applications," Echocardiography, vol. 26, no. 7, pp. 832-841, 2009.

[28] S. P. Hoole, P. M. Heck, L. Sharples et al., "Cardiac remote ischemic preconditioning in coronary stenting (CRISP Stent) study: a prospective, randomized control trial," Circulation, vol. 119, no. 6, pp. 820-827, 2009.

[29] H. M. C. Kemps, J. J. Prompers, B. Wessels et al., "Skeletal muscle metabolic recovery following submaximal exercise in chronic heart failure is limited more by $\mathrm{O}_{2}$ delivery than $\mathrm{O}_{2}$ utilization," Clinical Science, vol. 118, no. 3, pp. 203-210, 2010.

[30] M. Nakayama, S. Iwasaki, H. Ichinose, S. Yamamoto, N. Kanaya, and A. Namiki, "Monitoring of skeletal muscle oxygenation using near-infrared spectroscopy during abdominal aortic surgery," Journal of Anesthesia, vol. 16, no. 2, pp. 127-130, 2002.

[31] J. Sanders, I. S. Toor, T. M. Yurik, B. E. Keogh, M. Mythen, and H. E. Montgomery, "Tissue oxygen saturation and outcome after cardiac surgery," American Journal of Critical Care, vol. 20, no. 2, pp. 138-145, 2011.

[32] G. J. Kemp, N. Roberts, W. E. Bimson et al., "Mitochondrial function and oxygen supply in normal and in chronically ischemic muscle: a combined ${ }^{31} \mathrm{P}$ magnetic resonance spectroscopy and near infrared spectroscopy study in vivo," Journal of Vascular Surgery, vol. 34, no. 6, pp. 1103-1110, 2001.

[33] S. J. Thomson, M. L. Cowan, D. M. Forton et al., "A study of muscle tissue oxygenation and peripheral microcirculatory dysfunction in cirrhosis using near infrared spectroscopy," Liver International, vol. 30, no. 3, pp. 463-471, 2010.

[34] B. Grassi, M. Marzorati, F. Lanfranconi et al., "Impaired oxygen extraction in metabolic myopathies: detection and quantification by near-infrared spectroscopy," Muscle and Nerve, vol. 35, no. 4, pp. 510-520, 2007.

[35] M. Kawasuji, M. Ikeda, N. Sakakibara, S. Fujii, S. Tomita, and Y. Watanabe, "Near-infrared monitoring of myocardial oxygenation during ischemic preconditioning," Annals of Thoracic Surgery, vol. 69, no. 6, pp. 1806-1810, 2000.

[36] F. Thaveau, J. Zoll, O. Rouyer et al., "Ischemic preconditioning specifically restores complexes I and II activities of the mitochondrial respiratory chain in ischemic skeletal muscle," Journal of Vascular Surgery, vol. 46, no. 3, pp. 541-547, 2007.
[37] J. G. Kilian, S. Nakhla, K. Griffith, J. Harmer, M. Skilton, and D. S. Celermajer, "Reperfusion injury in the human forearm is mild and not attenuated by short-term ischaemic preconditioning," Clinical and Experimental Pharmacology and Physiology, vol. 32, no. 1-2, pp. 86-90, 2005.

[38] T. Hamaoka, H. Iwane, T. Shimomitsu et al., "Noninvasive measures of oxidative metabolism on working human muscles by near-infrared spectroscopy," Journal of Applied Physiology, vol. 81, no. 3, pp. 1410-1417, 1996.

[39] M. C. P. Van Beekvelt, W. N. J. M. Colier, R. A. Wevers, and B. G. M. Van Engelen, "Performance of near-infrared spectroscopy in measuring local $\mathrm{O}_{2}$ consumption and blood flow in skeletal muscle," Journal of Applied Physiology, vol. 90, no. 2, pp. 511-519, 2001.

[40] M. Ferrari, T. Binzoni, and V. Quaresima, "Oxidative metabolism in muscle," Philosophical Transactions of the Royal Society B, vol. 352, no. 1354 , pp. 677-683, 1997.

[41] S. Lavi and R. Lavi, "Conditioning of the heart: from pharmacological interventions to local and remote protection: possible implications for clinical practice," International Journal of Cardiology, vol. 146, no. 3, pp. 311-318, 2011. 

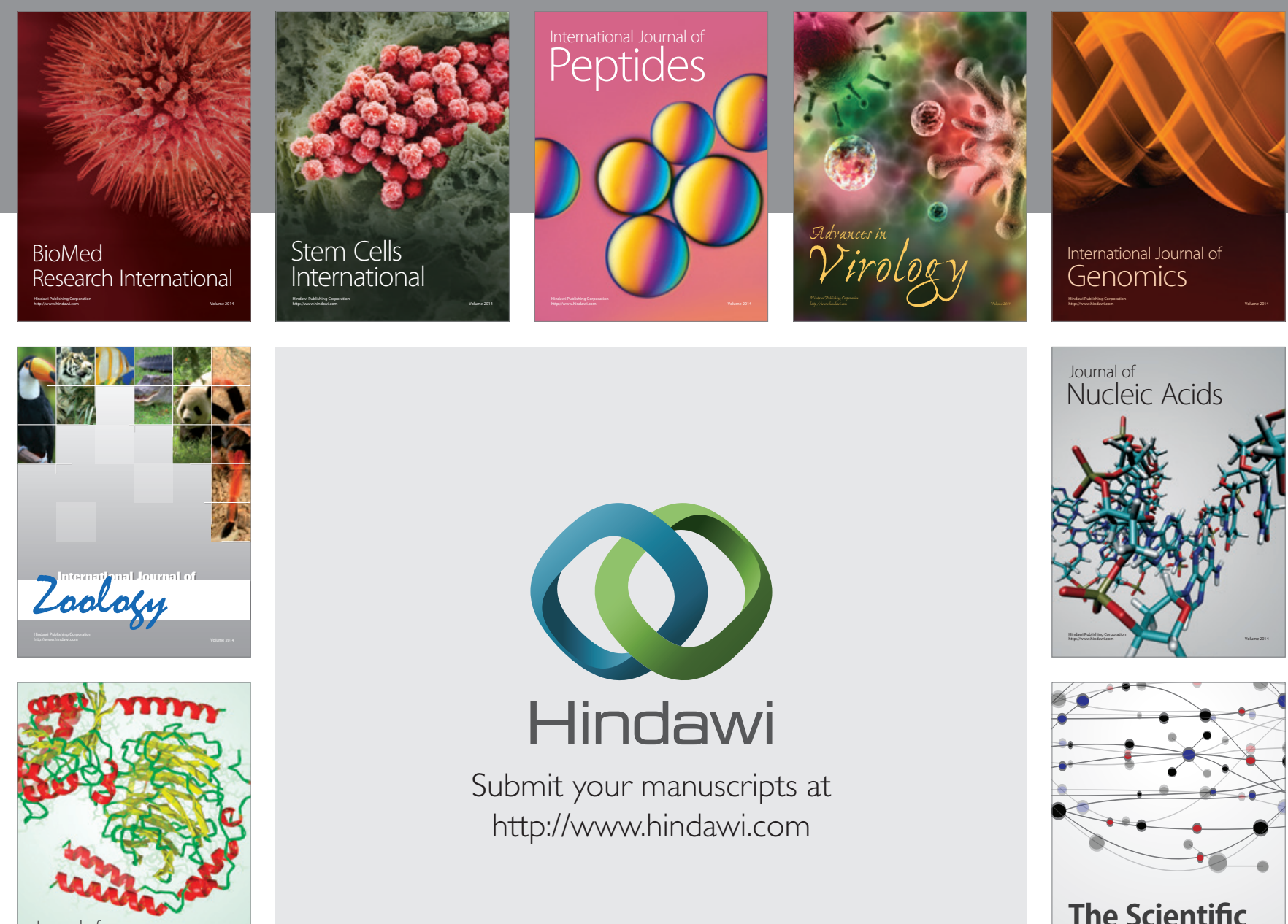

Submit your manuscripts at

http://www.hindawi.com

Journal of
Signal Transduction
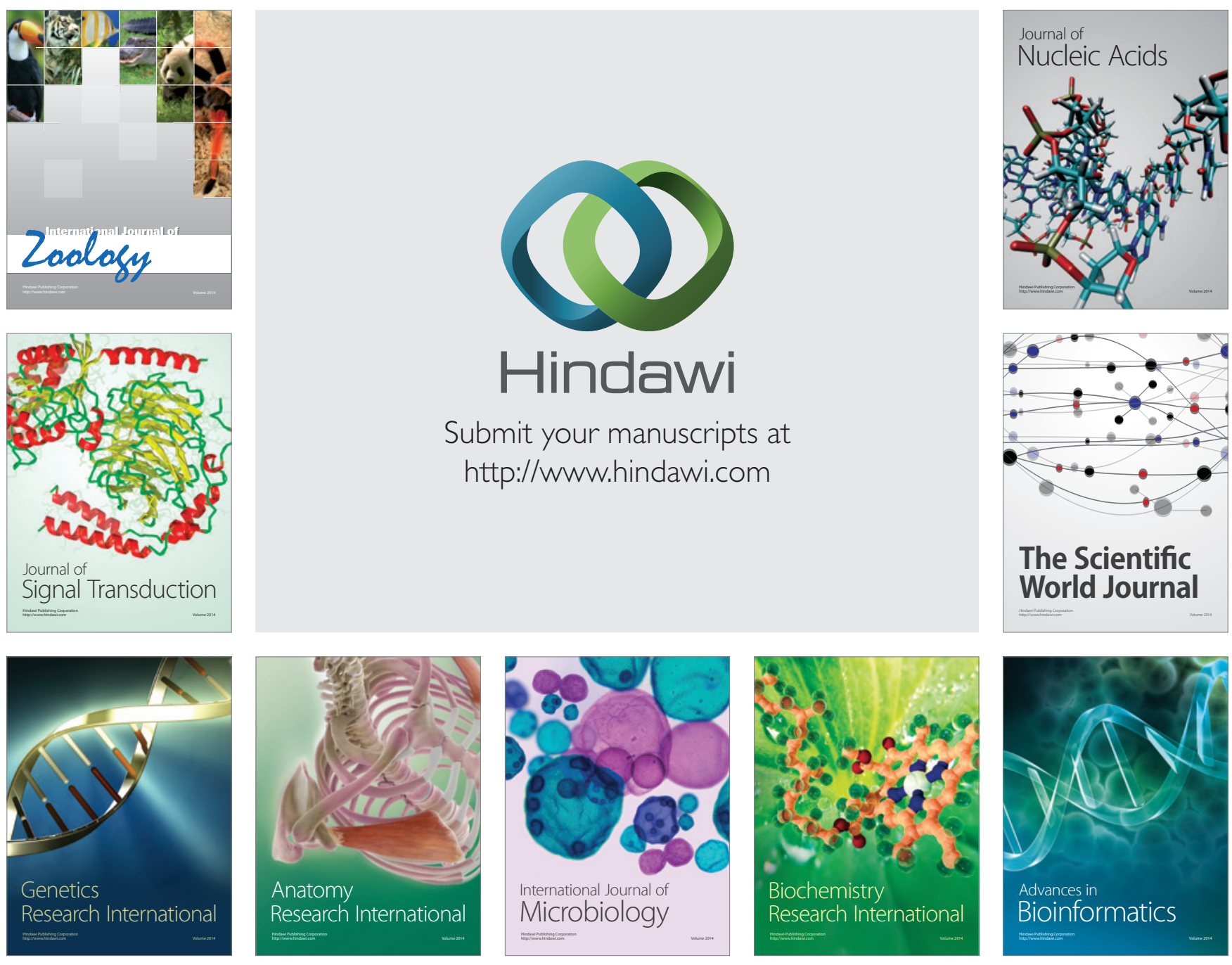

The Scientific World Journal
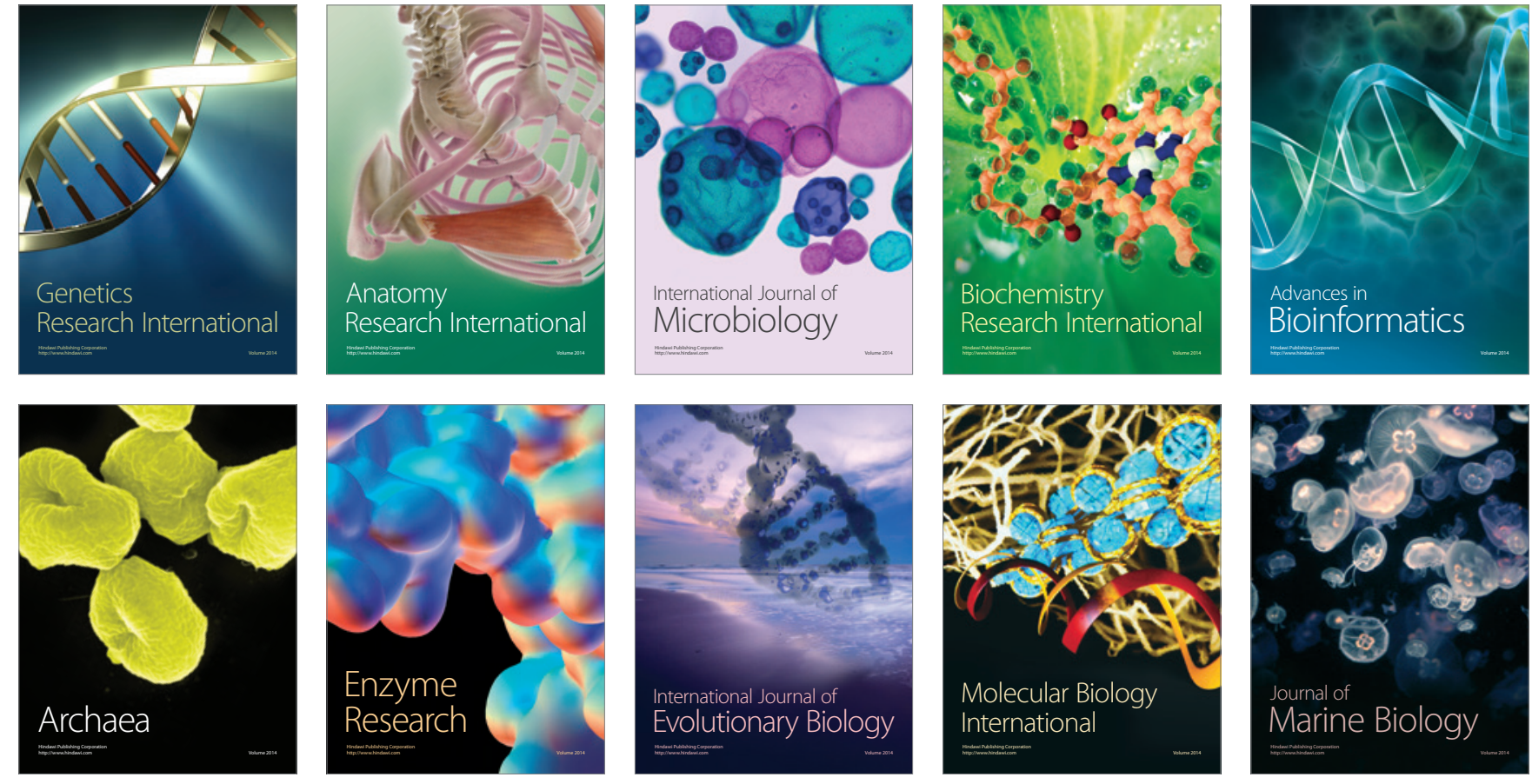